\title{
Effects of interphase coupling in one-dimensional particle-laden flow
}

\author{
Yahia M. Fouda ${ }^{1}$ iD
}

Received: 28 October 2020 / Accepted: 25 February 2021 / Published online: 12 March 2021

(c) The Author(s) $2021 \quad$ OPEN

\begin{abstract}
In Euler-Euler particle-laden flow models, the interphase coupling terms might include, in addition to the drag force, the effects of fluid pressure interaction with the particles and the volumetric displacement of the fluid phase due to the presence of the particles. This paper presents an analytical study on these effects for laminar, one-dimensional, steady, inviscid particle-laden flow. The interphase drag force is represented by a linear Stokes formula that is valid for low particle Reynolds number. It is shown mathematically that a full two-way coupling model, which includes all the aforementioned effects, depends on three parameters: inlet particle volume fraction (concentration), inlet density ratio-between the fluid and particle phases-and inlet velocity ratio. When the inlet particle concentration and density ratio are kept low at 0.01 and 0.001 , respectively, both the interphase pressure coupling force and the volumetric displacement of the fluid phase are insignificant. For density ratios of 0.001 and 1 and for the inlet particle concentration values examined, the effect of pressure interaction force is not significant. However, it becomes significant for higher density ratio of 10 at inlet particle concentration of 0.1 .
\end{abstract}

Keywords Particle-laden flow $\cdot$ Interphase coupling $\cdot$ Eulerian · One-dimensional flow

Mathematics Subject Classification $76 \mathrm{~T} 15 \cdot 83 \mathrm{C} 15$

\section{Introduction}

Particle-laden flows, in which a large number of small dispersed particles co-flow with liquid or gas, are ubiquitous in both engineering and natural processes. When the size of these particles is smaller than the smallest length scale of the relevant fluid motion, it is valid to approximate them as point-particles [1, 2]. This approximation allows the fluid phase to be described as a continuous media in the Eulerian framework. The dispersed particle phase, on the other hand, could be modelled using either the discrete Lagrangian approach [3-5] or the continuum Eulerian approach [6-9]. In the former, the equations of motion for each particle are solved in order to obtain its positions and velocities throughout its time of motion whereas in the latter, the particle phase is assumed to behave as a pseudo-fluid so that its variables are analogous to the hydrodynamic ones. The Lagrangian approach requires much more computational resources to calculate the trajectories and velocities of the massive number of particles encountered in realistic flow situations [10]. This led to extensive research effort in the development of Eulerian models [7, 11-16] and their application to different flow problems $[7,9,17-22]$.

In particle-laden flows, the continuum modelling of the fluid phase implies that the two-phases co-exist at every point in the domain while accounting for the fraction of volume occupied by each phase. Since the summation of the volume fractions of the two phases ought to be unity, the value of the volume fraction of either phase is used to formulate the governing equations because it determines how the two phases are coupled to each other. The oneway coupling model is often employed when the particle

$\triangle$ Yahia M. Fouda, Yahia_Fouda@mans.edu.eg|'Department of Mechanical Power Engineering, Faculty of Engineering, Mansoura University, Mansoura 35516, Egypt. 
volume fraction (concentration) is very low compared to that of the fluid phase $[6,8,9]$. This implies that the volume fraction of the fluid phase remains constant at unity. Furthermore, the effect of the hydrodynamic interactions between the two phases, for example the drag force, is negligible for the fluid phase but significant for the particle phase. Thus, one needs to solve the single phase flow equations for the fluid phase and subsequently, use the fluid flow field as an input parameter to obtain the particle phase flow field.

For moderate particle concentration, in which it is still valid to neglect the particle-particle interactions, a twoway interphase coupling approach is necessary. This is because the particles affect the fluid flow field so it cannot be evaluated independently. There are two differences between this two-way coupling model and the one-way coupling model. First, the hydrodynamic interactions have significant effects on the fluid phase. Second, the fluid phase volume fraction cannot be assumed constant at unity, which means that the volumetric displacement of the fluid by the particles should be accounted for [23]. However, in one class of the two-way coupling models, referred to as the dusty gas model [7], the fluid phase volume fraction is assumed constant at unity. This means that the fluid phase is coupled to the particle phase via the hydrodynamic interactions only. In either case, the twoway coupling models require the solution of the governing equations of the two phases simultaneously.

Owing to the complexity of the fluid flow governing equations and the infinite number of possible flow configurations, it is intuitive to claim that the transition from one-way coupling to two-way coupling is not universal. Even for a specific flow problem, it is not possible to determine quantitatively, prior to establishing the problem solution, the values of inlet or initial particle volume fraction, or other flow parameters, at which this transition takes place. The flow configuration could enrich some regions with particles and deplete other regions [23-25], resulting in different levels of interphase coupling across the domain. Thus, understanding the effect of interphase coupling requires solving the governing equations of the corresponding models for a specific flow problem then comparing their theoretical predictions. This approach has been used in previous research by employing the Lagrianian framework to simulate the particle phase using computational approaches [23-25]. The aim of this paper is, however, to use the Eulerian framework, for different levels of interphase coupling, in the description of a flow problem that can be solved using analytical methods. This analytical approach enables to elucidate the physics of the problem by understanding the functional relations in the models with different levels of interphase coupling.The rest of the paper is organised as follows. Section 2 presents the problem description and analytical solutions of four Eulerian models with different levels of interphase coupling. Section 3 presents some comparisons between the predictions of these models for a wide range of parameters. Section 4 summarises the paper findings highlighting its importance and limitations. Three appendices are used to explain some mathematical derivations of the analytical solutions.

\section{Problem description and analytical solution}

This paper considers a one-dimensional particle-laden flow problem in the streamwise direction with a uniform cross-sectional area. In order to focus on the effects of inertia and interphase coupling terms, the flow is assumed inviscid and the gravity effects are neglected for both phases. Furthermore, it is assumed that the slip velocity between the two phases is small, i.e. low particle Reynolds number, so that the linear Stokes drag relation is valid in describing the hydrodynamic interactions between the two phases. In what follows, these assumptions are applied to the governing equations of different Eulerian models representing various levels of interphase coupling in order to derive their analytical solutions.

\subsection{One-way coupling model}

The one-way coupling (1WC) model is used in the literature when the particle concentration (used interchangeably in this paper with "particle phase volume fraction") is too low to affect the gas phase $[6,9]$. Thus, the governing equations of the fluid phase are decoupled from that of the particle phase, which means they can be solved independently to obtain the fluid phase flow field. The particle phase, on the other hand, is coupled to the fluid phase so that its governing equations are [6]

$$
\begin{aligned}
& \frac{\partial}{\partial t}\left(\alpha \rho_{p}\right)+\frac{\partial}{\partial x_{j}}\left(\alpha \rho_{p} u_{p j}\right)=0, \\
& \frac{\partial}{\partial t}\left(\alpha \rho_{p} u_{p i}\right)+\frac{\partial}{\partial x_{j}}\left(\alpha \rho_{p} u_{p i} u_{p j}\right)=-\gamma \alpha \rho_{p}\left(u_{p i}-u_{f i}\right),
\end{aligned}
$$

where $\alpha$ is the particle phase volume fraction, $\rho_{p}$ is the particle material density, $u_{p i}$ is the particle phase velocity, $u_{f}$ is the fluid phase velocity, and $\gamma$ is the reciprocal of the particle relaxation time which is constant for low particle Reynolds number and given by $[6,9]$ 
$\gamma=\frac{18 \mu}{\rho_{p} d^{2}}$

where $\mu$ is the dynamic viscosity of the fluid and $d$ is the particle diameter. For one-dimensional steady flow, the continuity equation of the fluid phase implies that its velocity is constant at $U_{f}$ (the subscripts denoting the coordinates are dropped). Moreover, the continuity equation of the particle phase is

$\frac{\mathrm{d}}{\mathrm{d} x}\left(\alpha \rho_{p} u_{p}\right)=0$.

Thus, the non-conservative form of the particle phase momentum equation in the $x$ direction is

$u_{p} \frac{\mathrm{d} u_{p}}{\mathrm{~d} x}=-\gamma\left(u_{p}-U_{f}\right)$

In order to write Eq. (5) in the dimensionless form, where the star superscripts denote dimensionless variables, the following provisional scaling is introduced

$u_{p}=U_{p} u_{p}^{*}, x=X x^{*}$,

which is substituted in Eq. (5) to give

$\left(\frac{U_{p}}{\gamma X}\right) u_{p}^{*} \frac{\mathrm{d} u_{p}^{*}}{\mathrm{~d} x^{*}}=-\left(u_{p}^{*}-\frac{U_{f}}{U_{p}}\right)$

Defining the velocity ratio $B$ as

$B=\frac{U_{f}}{U_{p}}$,

where $U_{p}$ is the particle phase inlet velocity. By choosing the length scale $X$ as

$X=U_{p} / \gamma$,

the pre-factor of the left-hand side of equation (5) becomes unity. Thus, the dimensionless particle phase momentum equation now reads

$u_{p}{ }^{*} \frac{\mathrm{d} u_{p}{ }^{*}}{\mathrm{~d} x^{*}}=-\left(u_{p}^{*}-B\right)$.

Because the flow is one-dimensional, the particle phase continuity equation can be written as

$\alpha u_{p}=\alpha_{0} U_{p}$

where $\alpha_{0}$ is the inlet particle phase concentration which is used in the scaling as

$\alpha=\alpha_{0} \alpha^{*}$,

the continuity equation in the dimensionless form is thus $\alpha^{*} u_{p}^{*}=1$.

The non-dimensional particle phase momentum equation (10) can be integrated to obtain a solution for the dimensionless particle phase velocity [9]. Re-writing Eq. (10) as

$\left(\frac{u_{p}{ }^{*}}{u_{p}{ }^{*}-B}\right) \frac{\mathrm{d} u_{p}{ }^{*}}{\mathrm{~d} x^{*}}=-1$.

By using the separation of variables method and applying the boundary condition,

$u_{p}{ }^{*}(0)=1$,

the dimensionless particle phase velocity is given by the following implicit relation

$\left(u_{p}{ }^{*}-1\right)+B \ln \left(\frac{u_{p}{ }^{*}-B}{1-B}\right)=-x^{*}$.

Equation (16) can be inverted to obtain an explicit relation of the velocity in terms of Lambert $W$ function as [9]

$u_{p}^{*}=B\left[1+\mathrm{W}\left(\left(\frac{1-B}{B}\right) \exp \left(\frac{1-B-x^{*}}{B}\right)\right)\right]$.

The particle phase volume fraction can thus be obtained using the continuity equation (13). For fully developed flow, the particle phase velocity is obtained by setting $\mathrm{d} u_{p} / \mathrm{d} x=0$ in the momentum equation (10) resulting in

$u_{p}^{*}\left(x^{*} \rightarrow \infty\right)=B$.

\subsection{Dusty gas model}

The dusty gas (DG) model [7] uses a pseudo two-way coupling formulation. The particle phase momentum equation is the same as the one-way coupling model given by Eqs. (1) and (2). However, the particle phase affects the fluid phase via a momentum source term only, which makes the coupling between the phases two-way. Thus, one needs to solve the governing equations for the two phases simultaneously. These governing equations for the fluid phase are [7]

$\frac{\partial \rho_{f}}{\partial t}+\frac{\partial}{\partial x_{j}}\left(\rho_{f} u_{f j}\right)=0$,

$\frac{\partial}{\partial t}\left(\rho_{f} u_{f i}\right)+\frac{\partial}{\partial x_{j}}\left(\rho_{f} u_{f i} u_{f j}\right)=\gamma \alpha \rho_{p}\left(u_{p i}-u_{f i}\right)$

$+\frac{\partial}{\partial x_{i}}\left(-p \delta_{i j}+\tau_{i j}\right)$

where $\rho_{f}$ is the material density of the fluid phase, $p$ is the fluid phase pressure, $\delta_{i j}$ is the Kronecker delta, and $\tau_{i j}$ is the viscous stresses which is neglected in this paper. 
It should be noted that the continuity equation (19) of the fluid phase is identical to that of single phase flows, which results in a uniform fluid velocity for the one-dimensional incompressible flow problem under investigation. Thus, the momentum equation of the particle phase is the same as that of the one-way coupling model (5) resulting in the same particle velocity as (17). Moreover, the left-hand side of the fluid phase momentum equation is zero. Thus, the fluid phase momentum equation (20) becomes a function of the pressure only as

$\frac{\mathrm{d} p}{\mathrm{~d} x}=\gamma \alpha \rho_{p}\left(u_{p}-U_{f}\right)$.

Using the same scaling for the one-way coupling model, given by Eqs. (6) and (9), and a provisional scaling for the pressure, $p=p^{*} P_{0}$, where $P_{0}$ is a reference pressure, the momentum equation (21) in the dimensionless form is

$\left(\frac{P_{0}}{\alpha_{0} \rho_{p} U_{p}^{2}}\right) \frac{\mathrm{d} p^{*}}{\mathrm{~d} x^{*}}=\alpha^{*}\left(u_{p}{ }^{*}-B\right)$,

which shows that, for incompressible flow

$P_{0}=\alpha_{0} \rho_{p} U_{p}^{2}$

is a natural scaling of the fluid phase pressure, because it is divided by the particle phase inlet kinetic energy. Thus, the final dimensionless form of the fluid phase momentum equation is

$\frac{\mathrm{d} p^{*}}{\mathrm{~d} x^{*}}=\alpha^{*}\left(u_{p}^{*}-B\right)$.

It is possible to substitute (17) into (24) to obtain a differential equation describing the pressure as a function of the dimensionless distance $x^{*}$. However, it is more informative to use the particle phase velocity as the independent variable in the pressure Eq. (24). Thus, the chain rule is used to express the pressure gradient with respect to the particle phase velocity as

$\frac{\mathrm{d} p^{*}}{\mathrm{~d} x^{*}}=\frac{\mathrm{d} p^{*}}{\mathrm{~d} u_{p}^{*}} \frac{\mathrm{d} u_{p}^{*}}{\mathrm{~d} x^{*}}$.

By substituting Eqs. (13), (14) and (25) into Eq. (24), it can be shown that the variation of the pressure is constant with respect to the particle velocity as

$\frac{\mathrm{d} p^{*}}{\mathrm{~d} u_{p}{ }^{*}}=-1$

Using the boundary condition shown in Eq. (15) for the particle velocity and the following boundary condition for the pressure $p^{*}(0)=0$,

the solution is thus given by

$p^{*}=1-u_{p}^{*}$.

Note that the fluid pressure obtained here is different from that of the 1WC model because the latter is zero as its fluid flow velocity is constant. For fully developed flow, substitute Eq. (18) into Eq. (28) so that the pressure is

$p^{*}(x \rightarrow \infty)=1-B$.

\subsection{Two-way coupling model}

For a full two-way coupling (2WC) between the particle and the fluid phases [8], the continuity equation (1) of the particle phase, used in both the one-way coupling and the dusty gas model, remains intact. On the other hand, the momentum equation of the particle phase is given by [8]

$$
\begin{aligned}
& \frac{\partial}{\partial t}\left(\alpha \rho_{p} u_{p i}\right)+\frac{\partial}{\partial x_{j}}\left(\alpha \rho_{p} u_{p i} u_{p j}\right) \\
& =-\gamma \alpha \rho_{p}\left(u_{p i}-u_{f i}\right)-\alpha \frac{\partial}{\partial x_{i}}\left(p \delta_{i j}\right) .
\end{aligned}
$$

The governing equations for the fluid phase are given by [8]

$$
\frac{\partial}{\partial t}\left((1-\alpha) \rho_{f}\right)+\frac{\partial}{\partial x_{j}}\left((1-\alpha) \rho_{f} u_{f j}\right)=0,
$$

$\frac{\partial}{\partial t}\left((1-\alpha) \rho_{f} u_{f}\right)+\frac{\partial}{\partial x_{j}}\left((1-\alpha) \rho_{f} u_{f} u_{f j}\right)$

$=\gamma \alpha \rho_{p}\left(u_{p i}-u_{f i}\right)-(1-\alpha) \frac{\partial}{\partial x_{i}}\left(p \delta_{i j}\right)+\frac{\partial \tau_{i j}}{\partial x_{i}}$.

One difference between the 2WC model and the DG model is that the volumetric displacement of the fluid due to the presence of the particles is accounted for in the 2WC model. In the continuity equation (31) and the inertia terms on the left-hand side of the momentum equation (32), the fluid density is multiplied by the fluid phase volume fraction, $(1-\alpha)$. Furthermore, the two phase are coupled via a pressure interaction term, $\alpha \partial\left(p \delta_{i j}\right) / \partial x_{i}$. In one-dimension, the inviscid $2 W C$ model is

Particle phase continuity

$\frac{\mathrm{d}}{\mathrm{d} x}\left(\alpha \rho_{p} u_{p}\right)=0$.

Fluid phase continuity 
$\frac{\mathrm{d}}{\mathrm{d} x}\left((1-\alpha) \rho_{f} u_{f}\right)=0$

Particle phase momentum

$\frac{\mathrm{d}}{\mathrm{d} x}\left(\alpha \rho_{p} u_{p} u_{p}\right)=-\alpha \frac{\mathrm{d} p}{\mathrm{~d} x}-\gamma \rho_{p} \alpha\left(u_{p}-u_{f}\right)$.

Fluid phase momentum

$\frac{\mathrm{d}}{\mathrm{d} x}\left((1-\alpha) \rho_{f} u_{f} u_{f}\right)=-(1-\alpha) \frac{\mathrm{d} p}{\mathrm{~d} x}+\gamma \rho_{p} \alpha\left(u_{p}-u_{f}\right)$.

Integrating the particle phase continuity equation (34) gives

$\alpha \rho_{p} u_{p}=\alpha_{0} \rho_{p} U_{p}$.

Expanding the left-hand side of the particle phase momentum equation (35) and substituting the continuity equation (37), the particle phase momentum equation can be written as

$u_{p} \frac{\mathrm{d} u_{p}}{\mathrm{~d} x}=-\frac{1}{\rho_{p}} \frac{\mathrm{d} p}{\mathrm{~d} x}-\gamma\left(u_{p}-u_{f}\right)$.

Using the scaling defined previously as

$u_{p}=U_{p} u_{p}{ }^{*}, \quad x=U_{p} x^{*} / \gamma, u_{f}=U_{f} u_{f}{ }^{*}, p=\alpha_{0} \rho_{p} U_{p}{ }^{2} p^{*}$,

the dimensionless particle phase momentum equation is

$u_{p}{ }^{*} \frac{\mathrm{d} u_{p}^{*}}{\mathrm{~d} x^{*}}=-\alpha_{0} \frac{\mathrm{d} p^{*}}{\mathrm{~d} x^{*}}-\left(u_{p}^{*}-B u_{f}^{*}\right)$.

Expanding the left-hand side of the fluid phase momentum equation (36) $\frac{\mathrm{d} u_{f}^{*}}{\mathrm{~d} x^{*}}=A\left[-\left(1-\alpha_{0} \alpha^{*}\right) \frac{\mathrm{d} p^{*}}{\mathrm{~d} x^{*}}+\alpha^{*}\left(u_{p}^{*}-B u_{f}^{*}\right)\right]$,

$A=\left(\frac{\alpha_{0}}{1-\alpha_{0}}\right)\left(\frac{\rho_{p}}{\rho_{f}}\right)\left(\frac{U_{p}}{U_{f}}\right)^{2}$.

Applying the scaling given by (12) and (39) to the fluid phase continuity equation (34) results in the following dimensionless form

$\left(1-\alpha_{0} \alpha^{*}\right) u_{f}^{*}=\left(1-\alpha_{0}\right)$,

and the dimensionless continuity equation for the particle phase is the same as Eq. (13). Note that for the limit $\alpha_{0} \rightarrow 0$, the 2WC model, given by Eqs. (13), (40), (44) and (46), reduces to the DG model shown in Sect. 2.2.

In order to solve this $2 \mathrm{WC}$ model, the continuity equations of the two phases, (13) and (46), are used to obtain an algebraic relation between $u_{p}{ }^{*}$ and $u_{f}{ }^{*}$ by eliminating $\alpha^{*}$ as

$u_{f}^{*}=\left(1-\alpha_{0}\right)\left(\frac{u_{p}^{*}}{u_{p}{ }^{*}-\alpha_{0}}\right)$.

The first derivative of Eq. (47) is

$\frac{\mathrm{d} u_{f}^{*}}{\mathrm{~d} x^{*}}=-\frac{\alpha_{0}\left(1-\alpha_{0}\right)}{\left(u_{p}{ }^{*}-\alpha_{0}\right)^{2}}\left(\frac{\mathrm{d} u_{p}^{*}}{\mathrm{~d} x^{*}}\right)$.

Substituting $u_{f}{ }^{*}$, given by Eq. (47), into the particle phase momentum equation (40), the fluid phase pressure gradient is expressed as

$(1-\alpha) \rho_{f} u_{f} \frac{\mathrm{d} u_{f}}{\mathrm{~d} x}+u_{f} \frac{\mathrm{d}}{\mathrm{d} x}\left((1-\alpha) \rho_{f} u_{f}\right)=-(1-\alpha) \frac{\mathrm{d} p}{\mathrm{~d} x}+\gamma \rho_{p} \alpha\left(u_{p}-u_{f}\right)$.

By applying the continuity equation of the fluid phase (34), the second term on the left-hand side of Eq. (41) vanishes. Moreover, the continuity equation of the fluid phase is

$(1-\alpha) \rho_{f} u_{f}=\left(1-\alpha_{0}\right) \rho_{f} U_{f}$

thus, the momentum equation of the fluid phase now reads

$\left(1-\alpha_{0}\right) \rho_{f} U_{f} \frac{\mathrm{d} u_{f}}{\mathrm{~d} x}=-(1-\alpha) \frac{\mathrm{d} p}{\mathrm{~d} x}+\gamma \rho_{p} \alpha\left(u_{p}-u_{f}\right)$.

Using the scaling given by (12) and (39) and after some manipulation, the fluid phase dimensionless momentum equation is $\frac{\mathrm{d} p^{*}}{\mathrm{~d} x^{*}}=-\frac{1}{\alpha_{0}}\left(u_{p}{ }^{*} \frac{\mathrm{d} u_{p}{ }^{*}}{\mathrm{~d} x^{*}}+\frac{u_{p}{ }^{*}\left(u_{p}{ }^{*}-\alpha_{0}-B\left(1-\alpha_{0}\right)\right)}{\left(u_{p}{ }^{*}-\alpha_{0}\right)}\right)$.

Using the integrated continuity equation of the particle phase, $\alpha^{*}=1 / u_{p}{ }^{*}$, and Eq. (47), the fluid phase momentum (44) can be written as

$$
\begin{aligned}
\frac{\mathrm{d} p^{*}}{\mathrm{~d} x^{*}} & =-\frac{1}{A}\left(\frac{u_{p}{ }^{*}}{u_{p}{ }^{*}-\alpha_{0}}\right) \frac{\mathrm{d} u_{f}{ }^{*}}{\mathrm{~d} x^{*}} \\
& +\left(\frac{u_{p}^{*}}{u_{p}{ }^{*}-\alpha_{0}}\right)\left(1-\frac{B\left(1-\alpha_{0}\right)}{u_{p}{ }^{*}-\alpha_{0}}\right) .
\end{aligned}
$$

Substituting $\mathrm{d} u_{f}{ }^{*} / \mathrm{d} x^{*}$, given by Eq. (48), into Eq. (50), the following equation is obtained 
$\frac{\mathrm{d} p^{*}}{\mathrm{~d} x^{*}}=\frac{\alpha_{0}\left(1-\alpha_{0}\right) u_{p}{ }^{*}}{A\left(u_{p}{ }^{*}-\alpha_{0}\right)^{3}} \frac{\mathrm{d} u_{p}{ }^{*}}{\mathrm{~d} x^{*}}+\frac{u_{p}^{*}\left(u_{p}^{*}-\alpha_{0}-B\left(1-\alpha_{0}\right)\right)}{\left(u_{p}^{*}-\alpha_{0}\right)^{2}}$

Substituting $\mathrm{d} p^{*} / \mathrm{d} x^{*}$ given by Eq. (49), into Eq. (51), the following differential equation (see Appendix $A$ ) is obtained

$\frac{\mathrm{d} u_{p}{ }^{*}}{\mathrm{~d} x^{*}}=-\frac{A u_{p}{ }^{*}\left(u_{p}{ }^{*}-\alpha_{0}\right)\left(u_{p}{ }^{*}-\alpha_{0}-B\left(1-\alpha_{0}\right)\right)}{\alpha_{0}{ }^{2}\left(1-\alpha_{0}\right)+A\left(u_{p}{ }^{*}-\alpha_{0}\right)^{3}}$,

which is solved for $u_{p}{ }^{*}$ with the boundary condition $u_{p}{ }^{*}(0)=1$ using the separation of variables method (Appendix B) resulting in the following algebraic expression

$-\epsilon_{1} \ln u_{p}^{*}-\epsilon_{2} \ln \left(\frac{u_{p}^{*}-\alpha_{0}}{1-\alpha_{0}}\right)-\epsilon_{3} \ln \left(\frac{u_{p}^{*}-\alpha_{0}-D}{1-\alpha_{0}-D}\right)-\left(u_{p}^{*}-1\right)=x^{*}$,

where

$D=B\left(1-\alpha_{0}\right)$,

$\epsilon_{1}=\frac{D^{2} C-\alpha_{0}^{2}}{\alpha_{0}+D}$

$\epsilon_{2}=-D C$,

$\epsilon_{3}=\frac{D\left(\alpha_{0} C+D\right)}{\alpha_{0}+D}$

$C=\frac{\rho_{f}}{\rho_{p}}$.

Note that for the limit $\alpha_{0} \rightarrow 0$, the first two terms on the left-hand side of Eq. (53) cancel each other and $\epsilon_{3}=B$. Thus, the whole equation is identical to the solution of the DG model given by Eqs. (17) and (28). An explicit solution of the particle phase velocity requires inverting the left-hand of Eq. (53) which does not seem possible.

To derive the solution of the fluid phase pressure, the chain rule is applied to Eq. (49) in order to express the pressure gradient with respect to the particle phase velocity, in a similar fashion to Sect. 2.2, as

$\frac{\mathrm{d} p^{*}}{\mathrm{~d} u_{p}{ }^{*}} \frac{\mathrm{d} u_{p}^{*}}{\mathrm{~d} x^{*}}=-\frac{1}{\alpha_{0}}\left(u_{p}^{*} \frac{\mathrm{d} u_{p}^{*}}{\mathrm{~d} x^{*}}+\frac{u_{p}^{*}\left(u_{p}^{*}-\alpha_{0}-B\left(1-\alpha_{0}\right)\right)}{\left(u_{p}{ }^{*}-\alpha_{0}\right)}\right)$.

Substituting $\mathrm{d} u_{p}{ }^{*} / \mathrm{d} x^{*}$, given by Eq. (52), into Eq. (59) gives $\frac{\mathrm{d} p^{*}}{\mathrm{~d} u_{p}{ }^{*}}=-\frac{1}{\alpha_{0}}\left(u_{p}{ }^{*}-\frac{u_{p}{ }^{*}\left(u_{p}{ }^{*}-\alpha_{0}-B\left(1-\alpha_{0}\right)\right)\left(\alpha_{0}{ }^{2}\left(1-\alpha_{0}\right)+A\left(u_{p}{ }^{*}-\alpha_{0}\right)^{3}\right)}{A u_{p}{ }^{*}\left(u_{p}{ }^{*}-\alpha_{0}\right)^{2}\left(u_{p}{ }^{*}-\alpha_{0}-B\left(1-\alpha_{0}\right)\right)}\right)$,

which after some manipulation (Appendix C) and substituting $A$ from Eq. (45) becomes

$\frac{\mathrm{d} p^{*}}{\mathrm{~d} u_{p}{ }^{*}}=-1+\frac{C B^{2}\left(1-\alpha_{0}\right)^{2}}{\left(u_{p}{ }^{*}-\alpha_{0}\right)^{2}}$

then solving using the separation of variables method gives

$p^{*}=-u_{p}^{*}-\frac{\left(1-\alpha_{0}\right) C B^{2}}{\left(u_{p}{ }^{*}-\alpha_{0}\right)}+c$,

where $c$ is the constant of integration, which is evaluated using the boundary condition given by Eq. (27). Thus, the pressure field as a function of the particle phase velocity is

$p^{*}=\left(1-u_{p}^{*}\right)\left(1-\frac{\left(1-\alpha_{0}\right) C B^{2}}{\left(u_{p}^{*}-\alpha_{0}\right)}\right)$.

Note that the fluid pressure of the $2 \mathrm{WC}$ model equals to that of the DG model shown in Eq. (28) multiplied by $\left(1-C B^{2}\left(1-\alpha_{0}\right) /\left(u_{p}^{*}-\alpha_{0}\right)\right)$.

The fully developed particle phase velocity is obtained by setting its gradients to zero in Eq. (52) resulting in

$u_{p}{ }^{*}(x \rightarrow \infty)=B+\alpha_{0}(1-B)$,

which shows that the fully developed velocity of the $2 \mathrm{WC}$ equals to that of the 1WC and DG plus $\alpha_{0}(1-B)$. It is thus expected that the discrepancy between the $2 \mathrm{WC}$ and the $1 \mathrm{WC} / \mathrm{DG}$ increases with $\alpha_{0}$. The fully developed pressure is obtained by substituting Eq. (64) into Eq. (63) as

$p^{*}(x \rightarrow \infty)=(1-B)\left(1-\alpha_{0}\right)(1-B C)$,

which shows that the fully developed pressure of the $2 \mathrm{WC}$ equals to that of the DG model multiplied by $\left(1-\alpha_{0}\right)(1-B C)$. The fully developed fluid phase velocity is obtained by substituting Eq. (64) into Eq. (47) as

$u_{f}^{*}(x \rightarrow \infty)=1+\alpha_{0}\left(\frac{1}{B}-1\right)$,

which is equal to the inlet fluid phase velocity plus $\alpha_{0}(1 / B-1)$.

\subsection{Two-way coupling without pressure interaction}

In order to obtain an explicit solution for the particle phase velocity, $u_{p}{ }^{*}$, it is possible to approximate the $2 \mathrm{WC}$ by neglecting the pressure interaction term, $\alpha_{0}\left(d p^{*} / d x^{*}\right)$, 
in a model referred to as 2 WCNP. This approximation keeps the continuity equations of both phases intact, though it changes their momentum equations. For the fluid phase it becomes

$\frac{\mathrm{d} u_{f}^{*}}{\mathrm{~d} x^{*}}=A\left[-\frac{\mathrm{d} p^{*}}{\mathrm{~d} x^{*}}+\alpha^{*}\left(u_{p}^{*}-B u_{f}^{*}\right)\right]$,

and for the particle phase

$u_{p}{ }^{*} \frac{\mathrm{d} u_{p}{ }^{*}}{\mathrm{~d} x^{*}}=-\left(u_{p}^{*}-B u_{f}^{*}\right)$.

Substituting the expression of $u_{f}{ }^{*}$ from Eq. (47) into Eq. (68), the particle phase momentum equation contains $u_{p}{ }^{*}$ as the only dependent variable as

$\frac{\mathrm{d} u_{p}{ }^{*}}{\mathrm{~d} x^{*}}=-\frac{u_{p}{ }^{*}-\alpha_{0}-D}{u_{p}{ }^{*}-\alpha_{0}}$,

which can be written as

$\left(1+\frac{D}{u_{p}{ }^{*}-\alpha_{0}-D}\right) \frac{\mathrm{d} u_{p}^{*}}{\mathrm{~d} x^{*}}=-1$.

Using the separation of variables method and applying the boundary condition given by Eq. (15), the solution of Eq. (70) is

$\left(u_{p}{ }^{*}-1\right)+D \ln \left(\frac{u_{p}{ }^{*}-\alpha_{0}-D}{1-\alpha_{0}-D}\right)=-x^{*}$,

which has a similar form to Eq. (16). Thus, it can be shown that the explicit solution of the particle phase velocity is expressed in terms of Lambert $W$ function as

$u_{p}^{*}=\alpha_{0}+D\left[1+\mathrm{W}\left(\left(\frac{1-\alpha_{0}-D}{D}\right) \exp \left(\frac{1-\alpha_{0}-D-x^{*}}{D}\right)\right)\right]$.

Note that Eq. (72) reduces to Eq. (17) when $\alpha_{0} \rightarrow 0$, which is the underlying assumption of both the $1 \mathrm{WC}$ and DG models in neglecting the effect of particle phase volume fraction. The fluid phase velocity can be obtained by substituting Eq. (72) into Eq. (47).

For fully developed flow, the particle velocity is obtained by setting its gradient to zero in Eq. (69) to obtain

$u_{p}{ }^{*}(x \rightarrow \infty)=B+\alpha_{0}(1-B)$,

which is the same as the full two-way coupling model shown in Eq. (64).

In order to derive the solution for the pressure, substitute the continuity equation (13) and Eq. (47) into the momentum equation of the fluid phase (67) as $\frac{\mathrm{d} u_{f}^{*}}{\mathrm{~d} x^{*}}=-A \frac{\mathrm{d} p^{*}}{\mathrm{~d} x^{*}}+A\left(1-\frac{B\left(1-\alpha_{0}\right)}{\left(u_{p}{ }^{*}-\alpha_{0}\right)}\right)$.

Then substitute the value of $\mathrm{d} u_{f}{ }^{*} / \mathrm{d} x^{*}$ from Eq. (48) into Eq. (74) as

$-\left(\frac{\alpha_{0}\left(1-\alpha_{0}\right)}{A\left(u_{p}{ }^{*}-\alpha_{0}\right)^{2}}\right) \frac{\mathrm{d} u_{p}{ }^{*}}{\mathrm{~d} x^{*}}=-\frac{\mathrm{d} p^{*}}{\mathrm{~d} x^{*}}+\left(\frac{u_{p}{ }^{*}-\alpha_{0}-D}{u_{p}{ }^{*}-\alpha_{0}}\right)$.

According to Eq. (69), the last term on the right-hand side of Eq. (75) is $-\mathrm{d} u_{p}{ }^{*} / \mathrm{d} x^{*}$. Using the chain rule to express the pressure gradient with respect to $u_{p}{ }^{*}$, in a similar fashion to Sects. 2.2 and 2.3, Eq. (75) can be written as

$-\left(\frac{\alpha_{0}\left(1-\alpha_{0}\right)}{A\left(u_{p}{ }^{*}-\alpha_{0}\right)^{2}}\right) \frac{\mathrm{d} u_{p}^{*}}{\mathrm{~d} x^{*}}=-\frac{\mathrm{d} p^{*}}{\mathrm{~d} u_{p}{ }^{*}} \frac{\mathrm{d} u_{p}^{*}}{\mathrm{~d} x^{*}}-\frac{\mathrm{d} u_{p}^{*}}{\mathrm{~d} x^{*}}$,

which, after substituting $A$ from Eq. (45), results in the following differential equation describing the pressure as a derivative of the particle phase velocity

$\frac{\mathrm{d} p^{*}}{\mathrm{~d} u_{p}{ }^{*}}=-1+\frac{C B^{2}\left(1-\alpha_{0}\right)^{2}}{\left(u_{p}{ }^{*}-\alpha_{0}\right)^{2}}$.

which is identical to Eq. (61) of the $2 W C$ model. Thus, the fluid pressure of the 2WCNP is given by Eq. (63), and the fully developed fluid pressure is given by Eq. (65) because the fully developed velocity is the same as that of the 2WC. Moreover, since the fully developed particle phase velocity (73) and the continuity equation (47) are the same as the 2WC model, the fully developed gas phase velocity is the same as Eq. (66).

\section{Results and Discussion}

In what follows, the predictions of the dimensionless particle phase velocity and the fluid phase pressure are assessed using the analytical solutions of the models derived in Section 2 and summarised in Table 1. Because the particle phase velocity of the IWC is identical to that of the DG and the fluid phase pressure of the $1 \mathrm{WC}$ is constant at zero (inlet value), the 1WC model is not included in the following analysis. Thus, the focus here is on three models: DG, 2WC and 2WCNP. For the 2WC model, Table 1 shows that both the particle phase velocity and the fluid phase pressure depend on three dimensionless parameters: the inlet velocity ratio, $B$, the inlet particle concentration, $\alpha_{0}$, and the density ratio, $C$. The velocity obtained using 2WCNP does not depend on the density ratio, $C$, though its pressure depends on it. Both particle phase velocity 
Table 1 Summary of the models equations for the particle phase velocity and the fluid pressure

\begin{tabular}{lll}
\hline Method & $u_{p}{ }^{*}$ & $p^{*}$ \\
\hline 1WC & $B\left[1+\mathrm{W}\left(\left(\frac{1-B}{B}\right) \exp \left(\frac{1-B-x^{*}}{B}\right)\right)\right]$ & 0 \\
DG & $B\left[1+\mathrm{W}\left(\left(\frac{1-B}{B}\right) \exp \left(\frac{1-B-x^{*}}{B}\right)\right)\right]$ & $1-u_{p}{ }^{*}$ \\
2WC & Implicit form: Eq. $(53)$ & $\left(1-u_{p}{ }^{*}\right)\left(1-\frac{\left(1-\alpha_{0}\right) C B^{2}}{\left(u_{p}{ }^{*}-\alpha_{0}\right)}\right)$ \\
2WCNP & $\alpha_{0}+D\left[1+\mathrm{W}\left(\left(\frac{1-\alpha_{0}-D}{D}\right) \exp \left(\frac{1-\alpha_{0}-D-x^{*}}{D}\right)\right)\right]$ & $\left(1-u_{p}{ }^{*}\right)\left(1-\frac{\left(1-\alpha_{0}\right) C B^{2}}{\left(u_{p}^{*}-\alpha_{0}\right)}\right)$ \\
\hline
\end{tabular}

Table 2 Test cases for assessing the effects of problem parameters

\begin{tabular}{llll}
\hline Case number & $\begin{array}{l}\text { Velocity Ratio, } \\
B=U_{f} / U_{p}\end{array}$ & $\begin{array}{l}\text { Density Ratio, } \\
C=\rho_{f} / \rho_{p}\end{array}$ & $\alpha_{0}$ \\
\hline 1 & 2 & 0.001 & 0.01 \\
2 & 0.5 & 0.001 & 0.01 \\
3 & 2 & 0.001 & 0.1 \\
4 & 0.5 & 0.001 & 0.1 \\
5 & 2 & 1 & 0.01 \\
6 & 0.5 & 1 & 0.01 \\
7 & 2 & 1 & 0.1 \\
8 & 0.5 & 1 & 0.1 \\
9 & 2 & 10 & 0.01 \\
10 & 0.5 & 10 & 0.01 \\
11 & 2 & 10 & 0.1 \\
12 & 0.5 & 10 & 0.1 \\
\hline
\end{tabular}

and fluid phase pressure of the DG model depend on the velocity ratio, $B$, only.

In order to compare the aforementioned models over an applicable range of parameters, the predictions are obtained for 12 cases using the conditions shown in Table 2. For the density ratio, $0.001,1$ and 10 are used. For the particle concentration, 0.01 and 0.1 are used to represent particle flows of dilute and moderate concentration, respectively. The velocity ratio is kept small at 2 and 0.5 , because the underlying assumption of the model is low slip velocity between the two phases. The implicit form of the particle phase velocity for the $2 \mathrm{WC}$ is plotted by using the value of the velocity obtained from the 2WCNP model as the independent variable and calculating the dimensionless distance $x^{*}$ as the dependent variable.

Figures 1 and 2 show the particle phase velocity and the fluid phase pressure profiles, respectively, for a density ratio $C$ of 0.001 . In all cases and for all models, both velocity and pressure change with the distance until reaching the fully developed values. For a low particle concentration of 0.01 (cases 1 and 2), the velocity and pressure predictions of all three models are identical. This behaviour is expected because the DG model is a limiting case, where $\alpha_{0} \rightarrow 0$, of both 2WC and 2WCNP models.
When the particle concentration is increased to 0.1 (cases 3 and 4), the predictions of the 2WC and 2WCNP models remain identical, suggesting that the additional terms in Eq. (53) have negligible effects, for the low density ratio of 0.001 , compared to Eq. (72) or (71), even for high particle concentration of 0.1 . For cases 3 and 4 , the predictions of both 2WC and 2WCNP deviate from that of the DG model; suggesting that a value of inlet particle concentration of 0.1 is not small enough for the DG model to be accurate. For each case presented in Figs. 1 and 2, it is observed that the pressure trend is consistently opposite to that of the velocity; the fluid pressure decreases when the particle velocity increases and vice versa. This opposite sign of the velocity and pressure gradients is expected for the DG model; because $\mathrm{d} p^{*} / \mathrm{d} u_{p}{ }^{*}$ is always negative as shown in Eq. (26). For both 2WC and 2WCNP, Eq. (61), which is identical to Eq. (77), shows that $\mathrm{d} p^{*} / \mathrm{d} u_{p}{ }^{*}$ is negative when $C B^{2}\left(1-\alpha_{0}\right)^{2} /\left(u_{p}{ }^{*}-\alpha_{0}\right)^{2}<1$, which is expected because $C$ is very small at 0.001 .

Increasing the density ratio to 1 , as shown in Fig. 3, does not affect the behaviour of the velocity predictions of the three models, in a similar fashion to Fig. 1. All three models are identical for the low particle concentration of 0.01 , and the DG model deviates, for a particle concentration of 0.1 , from both the 2WC and 2WCNP.

Figure 4 shows the fluid phase pressure profiles for a density ratio of 1 . For both values of particle concentration (0.01 and 0.1), there is a significant discrepancy between the predictions of the DG model and that of the $2 \mathrm{WC} / 2 \mathrm{WCNP}$ models. When $B=2$ (cases 5 and 7 ), the pressure trends of DG and $2 \mathrm{WC} / 2 \mathrm{WCNP}$ are opposite, with the former decreasing monotonically and the latter increasing monotonically. This is because the term $C B^{2}\left(1-\alpha_{0}\right)^{2} /\left(u_{p}{ }^{*}-\alpha_{0}\right)^{2}$ in Eq. (61), which dictates the pressure sign, becomes greater than unity for the relatively large density ratio, $C=1$. This makes the fluid pressure gradient with respect to the particle velocity positive, i.e. they have the same trend. When $B=0.5$ (cases 6 and 8 ), there is a discrepancy between the 2WC/2WCNP and the DG, though all three models have the same increasing trend which is opposite to that of the particle velocity. This is because the term $C B^{2}\left(1-\alpha_{0}\right)^{2} /\left(u_{p}{ }^{*}-\alpha_{0}\right)^{2}$ in Eq. 

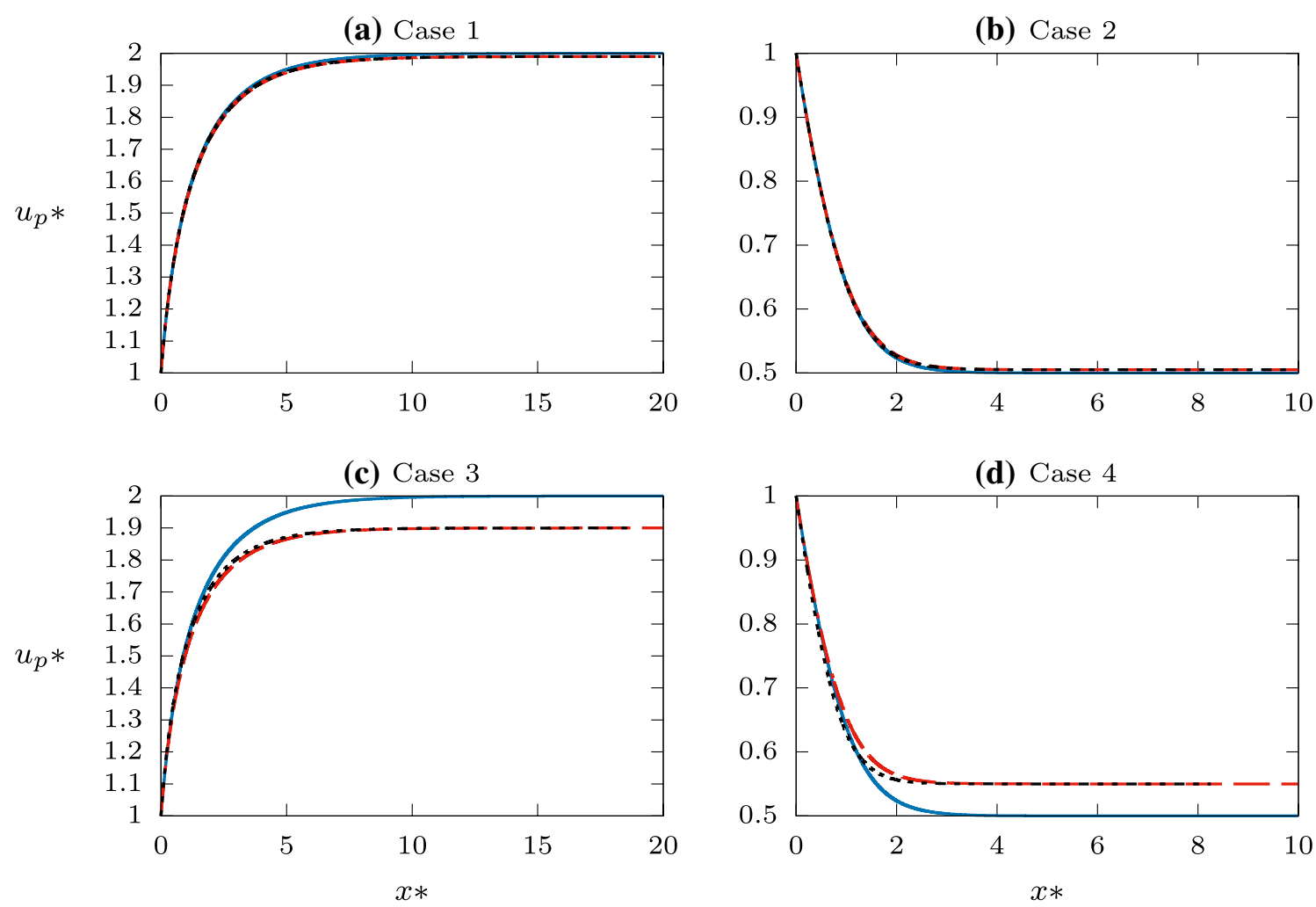

Fig. 1 Comparison between the velocity predictions of different models at $C=0.001$ : DG (blue solid lines); 2WCNP (red dashed lines); 2 WC (black dotted lines)

(61) become less than unity, because $B^{2}$ is small at 0.025 compared to 16 in cases 5 and 7 .

Figures 5 and 6 show the velocity and pressure predictions, respectively, using a density ratio $C$ of 10 . The velocity predictions of all models are nearly identical, when using a low inlet particle concentration of 0.01 , as cases 9 and 10 in Fig. 5 show. In contrast to the previous figures, there is a pronounced discrepancy, for inlet particle concentration of 0.1 , in the velocity predictions of the 2WC model compared to that of the 2WCNP model, as cases 11 and 12 in Fig. 5 show. This suggests that the additional terms of Eq. (53) compared to Eqs. (71) or (72) cannot be neglected for this value of density ratio. For an inlet particle concentration of 0.01 and velocity ratio of 2 , the pressure predictions of both the $2 \mathrm{WC}$ and $2 \mathrm{WCNP}$ are identical, and they deviate from that of the DG model, as shown in case 9 of Fig. 6 . Here, the discrepancy between the $2 \mathrm{WC} / 2 \mathrm{WCNP}$ and the DG is greater than that of case 5 shown in Fig. 4. This is because, for both the 2WC and 2WCNP models, the pressure is a function of the density ratio $C$, as shown by Eq. (63), whereas the pressure of the DG model does not depend on C, as shown in Eq. (28). In case 10 of Fig. 6 , where the velocity ratio is 0.5 , the discrepancy between 2 WC/2WCNP and DG is also clear. A main difference in the $2 \mathrm{WC} / 2 \mathrm{WCNP}$ model predictions here, compared to case 6 of Fig. 4, where the density ratio is 1 , is that in the former the pressure decreases whereas in the latter it increases. Again, this is traced to the additional term in Eqs. (61) and (77), $C B^{2}\left(1-\alpha_{0}\right)^{2} /\left(u_{p}{ }^{*}-\alpha_{0}\right)^{2}$, which becomes greater than unity for large $C$, making the pressure gradient with respect to the particle velocity positive. In a similar fashion to the velocity profiles shown in cases 11 and 12 of Fig. 5, there is a pronounced discrepancy between the pressure predictions when using higher inlet particle concentration of 0.1 in cases 11 and 12 of Fig. 6 . This is because, despite having identical pressure equations for both the 2WC and 2WCNP models, the pressure is a function of the velocity, which depends on $C$ for the $2 W C$ model and does not depend on $C$ for the 2WCNP model.

\section{Conclusions}

This paper presented an analytical study concerning the effects of the interphase coupling on the predictions of onedimensional steady particle-laden flow models in the Eulerian formulation. Four models involving different levels of interphase coupling were analysed: one-way coupling (1WC), 

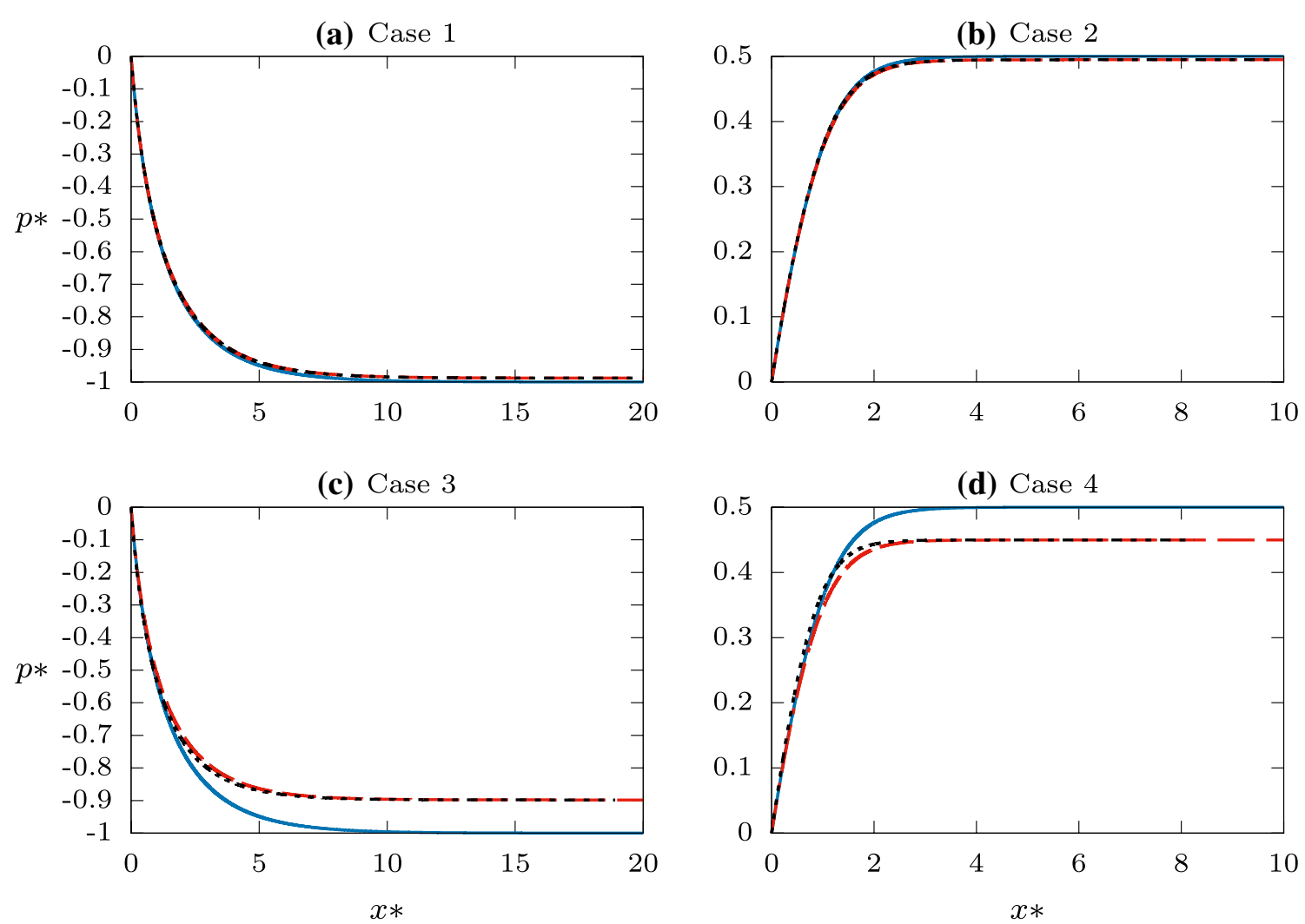

Fig. 2 Comparison between the pressure predictions of different models at $C=0.001$ : DG (blue solid lines); 2 WCNP (red dashed lines); 2 WC (black dotted lines)

dusty gas (DG), two-way coupling with (2WC) and without (2WCNP) interphase pressure interaction. It was shown mathematically that the $2 \mathrm{WC}$ model depends on three parameters: the inlet particle volume fraction, inlet density and velocity ratios between the fluid and particle phases. Because the DG model is a special case of the $2 \mathrm{WC} / 2 \mathrm{WCNP}$, where the inlet particle concentration vanishes, its predictions are identical to that of the $2 \mathrm{WC} / 2 \mathrm{WCNP}$ when the inlet particle concentration and density ratio are kept low at 0.01 and 0.001 , respectively. This confirms that both the interphase pressure coupling force and the volumetric displacement of the fluid phase are insignificant for these values. For density ratios of 0.001 and 1 and for all particle concentrations examined, the effect of pressure coupling force, which was evident by comparing the $2 \mathrm{WC}$ and $2 \mathrm{WCNP}$, is not significant. It becomes significant, however, for higher density ratio of 10 at inlet particle concentration of 0.1.The flow problem studied in this paper is fairly simple-realistic particle-laden laminar flows are often multi-dimensional with more complicated forms of interphase interactions, i.e. the drag force is not within the Stokes regime. However, it is necessary to study such simple configuration using analytical methodology as it provides some understanding of more complicated geometries and physics.

\section{Declarations}

Conflict of interest The author declares that he has no conflict of interest

Open Access This article is licensed under a Creative Commons Attribution 4.0 International License, which permits use, sharing, adaptation, distribution and reproduction in any medium or format, as long as you give appropriate credit to the original author(s) and the source, provide a link to the Creative Commons licence, and indicate if changes were made. The images or other third party material in this article are included in the article's Creative Commons licence, unless indicated otherwise in a credit line to the material. If material is not included in the article's Creative Commons licence and your intended use is not permitted by statutory regulation or exceeds the permitted use, you will need to obtain permission directly from the copyright holder. To view a copy of this licence, visit http://creativecommons .org/licenses/by/4.0\%. 

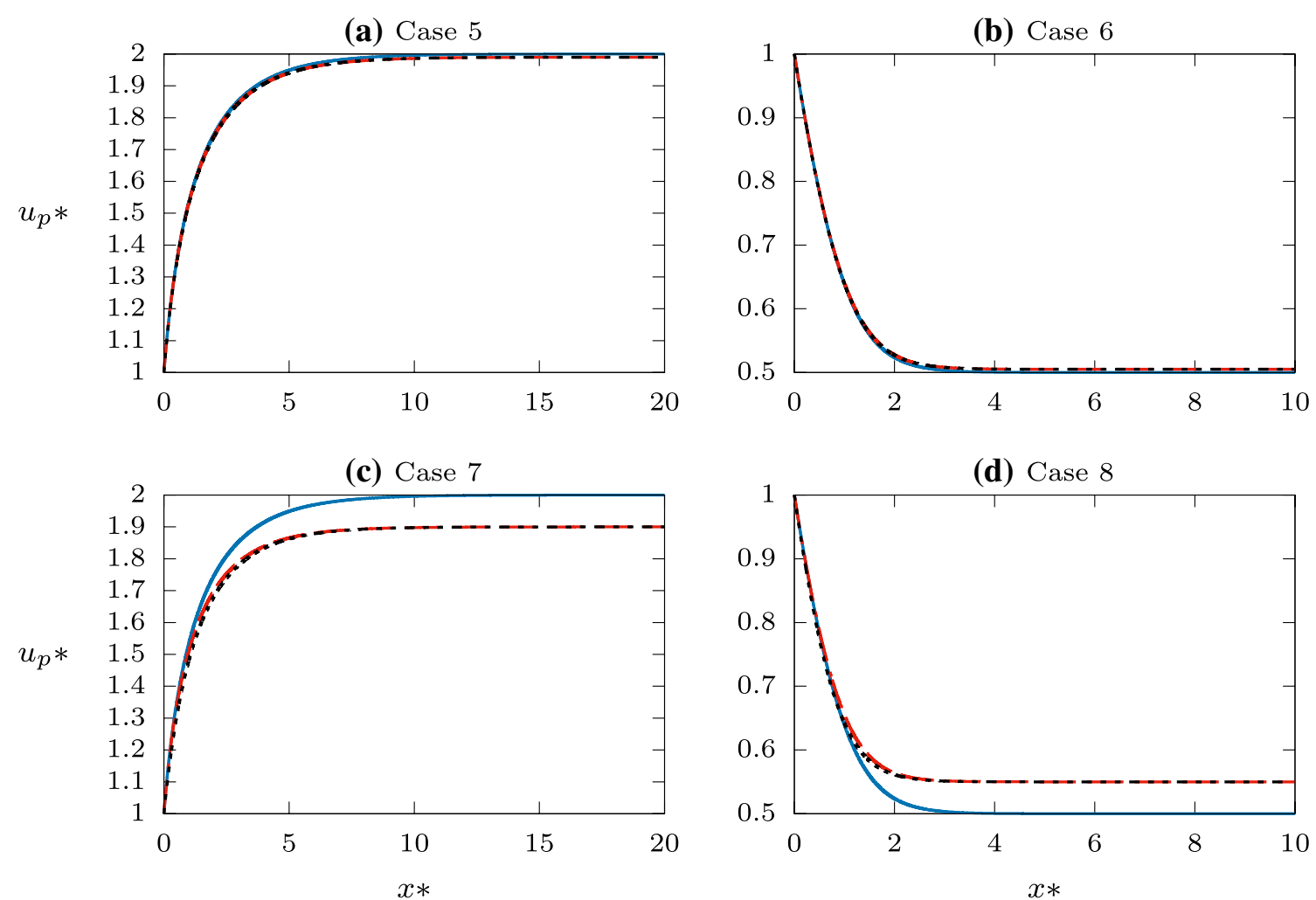

Fig. 3 Comparison between the velocity predictions of different models at $C=1.0$ : DG (blue solid lines); 2 WCNP (red dashed lines); 2 WC (black dotted lines)

\section{Appendix A}

In order to derive Eq. (52), substitute $\mathrm{d} p^{*} / \mathrm{d} x^{*}$ from Eq. (49) into Eq. (51) as

$$
\begin{aligned}
& \frac{1}{\alpha_{0}}\left[u_{p}{ }^{*} \frac{\mathrm{d} u_{p}^{*}}{\mathrm{~d} x^{*}}+\frac{u_{p}{ }^{*}\left(u_{p}{ }^{*}-\alpha_{0}-B\left(1-\alpha_{0}\right)\right)}{\left(u_{p}{ }^{*}-\alpha_{0}\right)}\right] \\
& =\frac{\alpha_{0}\left(1-\alpha_{0}\right) u_{p}{ }^{*}}{A\left(u_{p}{ }^{*}-\alpha_{0}\right)^{3}} \frac{\mathrm{d} u_{p}{ }^{*}}{\mathrm{~d} x^{*}}+\frac{u_{p}{ }^{*}\left(u_{p}{ }^{*}-\alpha_{0}-B\left(1-\alpha_{0}\right)\right)}{\left(u_{p}{ }^{*}-\alpha_{0}\right)^{2}} .
\end{aligned}
$$

$$
\begin{aligned}
{\left[1+\frac{\alpha_{0}{ }^{2}\left(1-\alpha_{0}\right)}{A\left(u_{p}{ }^{*}-\alpha_{0}\right)^{3}}\right] \frac{\mathrm{d} u_{p}^{*}}{\mathrm{~d} x^{*}} } & -\frac{\left(u_{p}^{*}-\alpha_{0}-B\left(1-\alpha_{0}\right)\right)}{\left(u_{p}{ }^{*}-\alpha_{0}\right)} \\
= & \frac{\alpha_{0}\left(u_{p}{ }^{*}-\alpha_{0}-B\left(1-\alpha_{0}\right)\right)}{\left(u_{p}{ }^{*}-\alpha_{0}\right)^{2}},
\end{aligned}
$$

Multiply Eq. (A1) by $-\alpha_{0} / u_{p}{ }^{*}$,

$\frac{\mathrm{d} u_{p}{ }^{*}}{\mathrm{~d} x^{*}}-\frac{\left(u_{p}{ }^{*}-\alpha_{0}-B\left(1-\alpha_{0}\right)\right)}{\left(u_{p}{ }^{*}-\alpha_{0}\right)}=\frac{\alpha_{0}{ }^{2}\left(1-\alpha_{0}\right)}{A\left(u_{p}{ }^{*}-\alpha_{0}\right)^{3}} \frac{\mathrm{d} u_{p}{ }^{*}}{\mathrm{~d} x^{*}}+\frac{\alpha_{0}\left(u_{p}{ }^{*}-\alpha_{0}-B\left(1-\alpha_{0}\right)\right)}{\left(u_{p}{ }^{*}-\alpha_{0}\right)^{2}}$.

Arrange similar terms of Eq. (A2)

$$
\begin{aligned}
& {\left[\frac{A\left(u_{p}^{*}-\alpha_{0}\right)^{3}+\alpha_{0}{ }^{2}\left(1-\alpha_{0}\right)}{A\left(u_{p}{ }^{*}-\alpha_{0}\right)^{3}}\right] \frac{\mathrm{d} u_{p}^{*}}{\mathrm{~d} x^{*}}} \\
& =-\frac{\left(u_{p}^{*}-\alpha_{0}-B\left(1-\alpha_{0}\right)\right)}{\left(u_{p}{ }^{*}-\alpha_{0}\right)}\left(1+\frac{\alpha_{0}}{u_{p}{ }^{*}-\alpha_{0}}\right),
\end{aligned}
$$


(a) Case 5

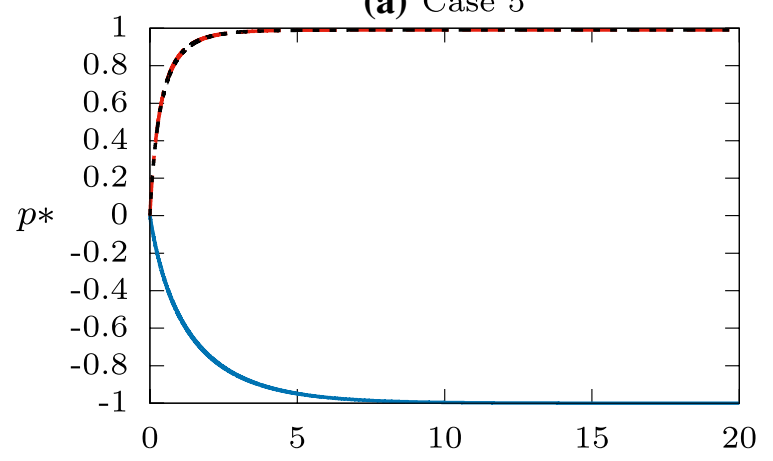

(c) Case 7

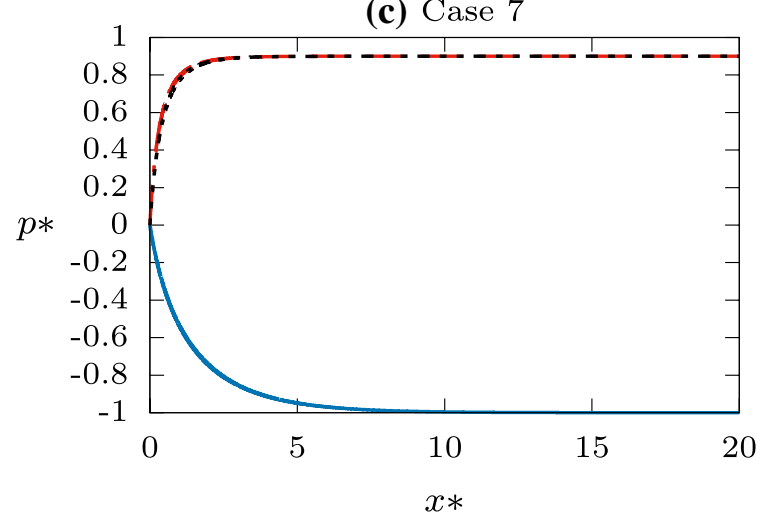

(b) Case 6

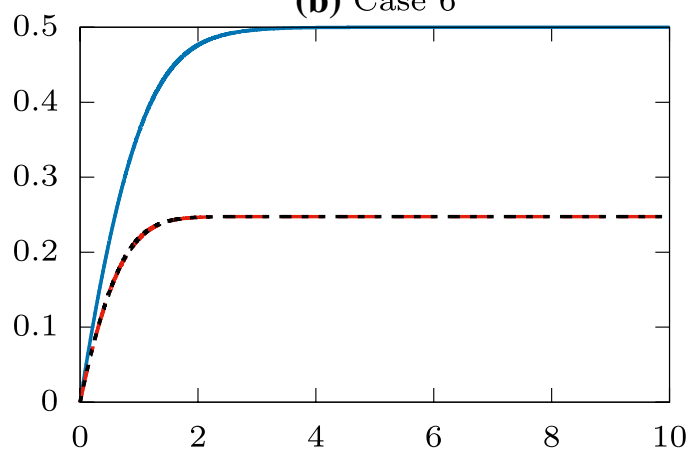

(d) Case 8

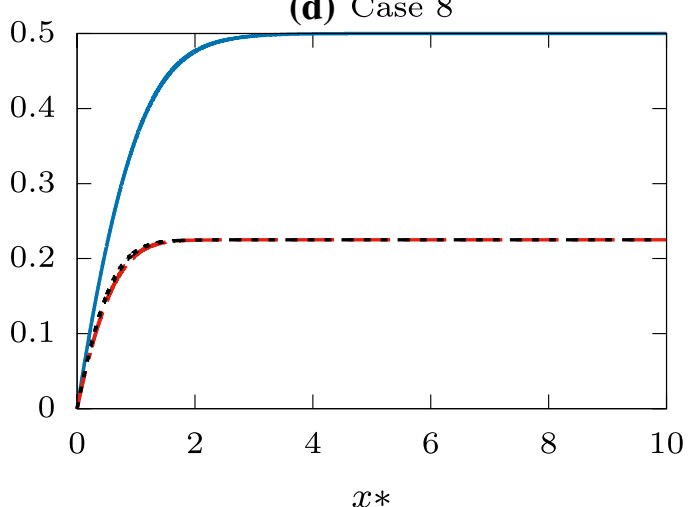

Fig. 4 Comparison between the pressure predictions of different models at $C=1.0$ : DG (blue solid lines); 2WCNP (red dashed lines); 2WC (black dotted lines)

$$
\begin{aligned}
& {\left[\frac{A\left(u_{p}{ }^{*}-\alpha_{0}\right)^{3}+\alpha_{0}{ }^{2}\left(1-\alpha_{0}\right)}{A\left(u_{p}{ }^{*}-\alpha_{0}\right)^{3}}\right] \frac{d u_{p}{ }^{*}}{d x^{*}}} \\
& \quad=-\frac{\left(u_{p}{ }^{*}-\alpha_{0}-B\left(1-\alpha_{0}\right)\right)}{\left(u_{p}{ }^{*}-\alpha_{0}\right)}\left(\frac{u_{p}{ }^{*}}{u_{p}{ }^{*}-\alpha_{0}}\right),
\end{aligned}
$$

$$
\begin{gathered}
{\left[\frac{A\left(u_{p}{ }^{*}-\alpha_{0}\right)^{3}+\alpha_{0}{ }^{2}\left(1-\alpha_{0}\right)}{A\left(u_{p}{ }^{*}-\alpha_{0}\right)^{3}}\right] \frac{\mathrm{d} u_{p}{ }^{*}}{\mathrm{~d} x^{*}}} \\
=-\frac{u_{p}{ }^{*}\left(u_{p}{ }^{*}-\alpha_{0}-B\left(1-\alpha_{0}\right)\right)}{\left(u_{p}{ }^{*}-\alpha_{0}\right)^{2}} .
\end{gathered}
$$

Then it is straightforward to derive Eq. (52) from Eq. (A6).

\section{Appendix B}

In order to solve the particle phase velocity for the twoway coupling model, write Eq. (52) as

$$
\left(\frac{\alpha_{0}{ }^{2}\left(1-\alpha_{0}\right)+A\left(u_{p}{ }^{*}-\alpha_{0}\right)^{3}}{A u_{p}{ }^{*}\left(u_{p}{ }^{*}-\alpha_{0}\right)\left(u_{p}{ }^{*}-\alpha_{0}-B\left(1-\alpha_{0}\right)\right)}\right) \mathrm{d} u_{p}{ }^{*}=-\mathrm{d} x^{*} .
$$

Then manipulate Eq. (B1) as

$$
\begin{aligned}
& {\left[\frac{\alpha_{0}{ }^{2}\left(1-\alpha_{0}\right)}{A u_{p}{ }^{*}\left(u_{p}{ }^{*}-\alpha_{0}\right)\left(u_{p}{ }^{*}-\alpha_{0}-D\right)}\right.} \\
& \left.+\frac{A\left(u_{p}{ }^{*}-\alpha_{0}\right)^{3}}{A u_{p}{ }^{*}\left(u_{p}{ }^{*}-\alpha_{0}\right)\left(u_{p}{ }^{*}-\alpha_{0}-D\right)}\right] \\
& \mathrm{d} u_{p}{ }^{*}=-\mathrm{d} x^{*},
\end{aligned}
$$

$$
\left[\frac{\alpha_{0}^{2}\left(1-\alpha_{0}\right)}{A u_{p}{ }^{*}\left(u_{p}{ }^{*}-\alpha_{0}\right)\left(u_{p}{ }^{*}-\alpha_{0}-D\right)}\right.
$$

$$
\left.+\frac{\left(u_{p}^{*}-\alpha_{0}\right)^{2}}{u_{p} *\left(u_{p}{ }^{*}-\alpha_{0}-D\right)}\right]
$$

$\mathrm{d} u_{p}{ }^{*}=-\mathrm{d} x^{*}$, 

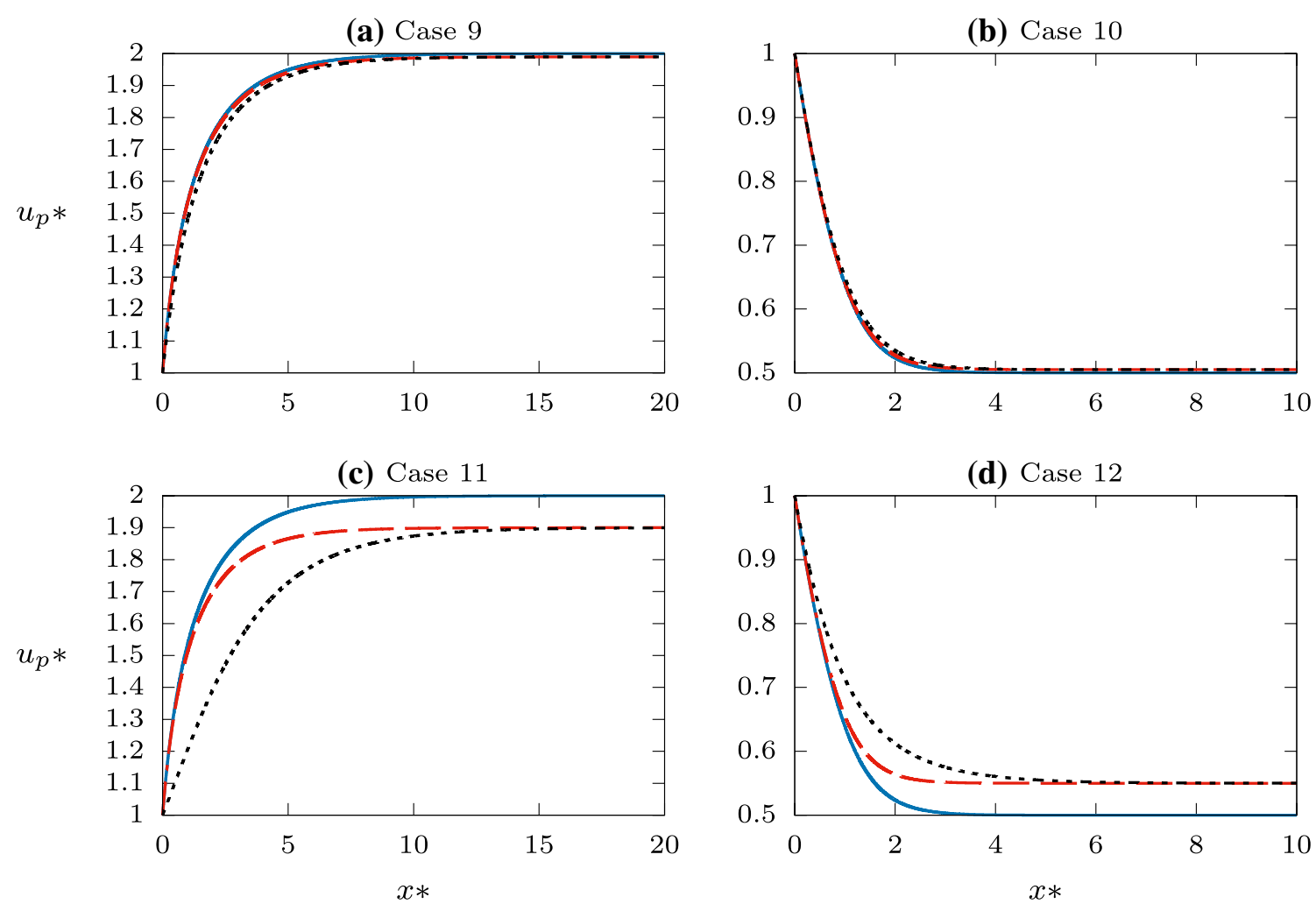

Fig. 5 Comparison between the velocity predictions of different models at $C=10.0$ : DG (blue solid lines); 2 WCNP (red dashed lines); 2 WC (black dotted lines)

$$
\begin{aligned}
& {\left[\frac{\alpha_{0}{ }^{2}\left(1-\alpha_{0}\right)}{A u_{p}{ }^{*}\left(u_{p}{ }^{*}-\alpha_{0}\right)\left(u_{p}{ }^{*}-\alpha_{0}-D\right)}\right.} \\
& \left.+\frac{\left(u_{p}{ }^{*}-\alpha_{0}\right)^{2}-D^{2}+D^{2}}{u_{p}{ }^{*}\left(u_{p}{ }^{*}-\alpha_{0}-D\right)}\right] \mathrm{d} u_{p}{ }^{*}=-\mathrm{d} x^{*}, \\
& {\left[\frac{\alpha_{0}{ }^{2}\left(1-\alpha_{0}\right)}{A u_{p}{ }^{*}\left(u_{p}{ }^{*}-\alpha_{0}\right)\left(u_{p}{ }^{*}-\alpha_{0}-D\right)}\right.} \\
& \left.+\frac{\left(u_{p}{ }^{*}-\alpha_{0}-D\right)\left(u_{p}{ }^{*}-\alpha_{0}+D\right)+D^{2}}{u_{p}{ }^{*}\left(u_{p}{ }^{*}-\alpha_{0}-D\right)}\right]
\end{aligned}
$$$$
\mathrm{d} u_{p}^{*}=-\mathrm{d} x^{*},
$$$$
\left[\frac{\alpha_{0}{ }^{2}\left(1-\alpha_{0}\right)}{A u_{p}{ }^{*}\left(u_{p}{ }^{*}-\alpha_{0}\right)\left(u_{p}{ }^{*}-\alpha_{0}-D\right)}+\frac{u_{p}{ }^{*}-\alpha_{0}+D}{u_{p}{ }^{*}}\right.
$$$$
\left.+\frac{D^{2}}{u_{p}^{*}\left(u_{p}^{*}-\alpha_{0}-D\right)}\right]
$$$$
\mathrm{d} u_{p}{ }^{*}=-\mathrm{d} x^{*},
$$

$$
\left[\frac{\alpha_{0}{ }^{2}\left(1-\alpha_{0}\right)}{A u_{p}{ }^{*}\left(u_{p}{ }^{*}-\alpha_{0}\right)\left(u_{p}{ }^{*}-\alpha_{0}-D\right)}+1-\frac{\alpha_{0}}{u_{p}{ }^{*}}\right.
$$$$
\left.+\frac{D}{u_{p}{ }^{*}}+\frac{D^{2}}{u_{p}{ }^{*}\left(u_{p}{ }^{*}-\alpha_{0}-D\right)}\right]
$$

$\mathrm{d} u_{p}{ }^{*}=-\mathrm{d} x^{*}$.

let

$R_{1}=\frac{\alpha_{0}{ }^{2}\left(1-\alpha_{0}\right)}{A u_{p}{ }^{*}\left(u_{p}{ }^{*}-\alpha_{0}\right)\left(u_{p}{ }^{*}-\alpha_{0}-D\right)}$,

$R_{2}=\frac{D^{2}}{u_{p} *\left(u_{p}^{*}-\alpha_{0}-D\right)}$.

Then Eq. (B7) can be written as

$\left[R_{1}+\left(1-\frac{\alpha_{0}}{u_{p}{ }^{*}}+\frac{D}{u_{p}{ }^{*}}\right)+R_{2}\right] \mathrm{d} u_{p}^{*}=-\mathrm{d} x^{*}$.

Using partial fraction decomposition, Eq. (B8) can be written as 

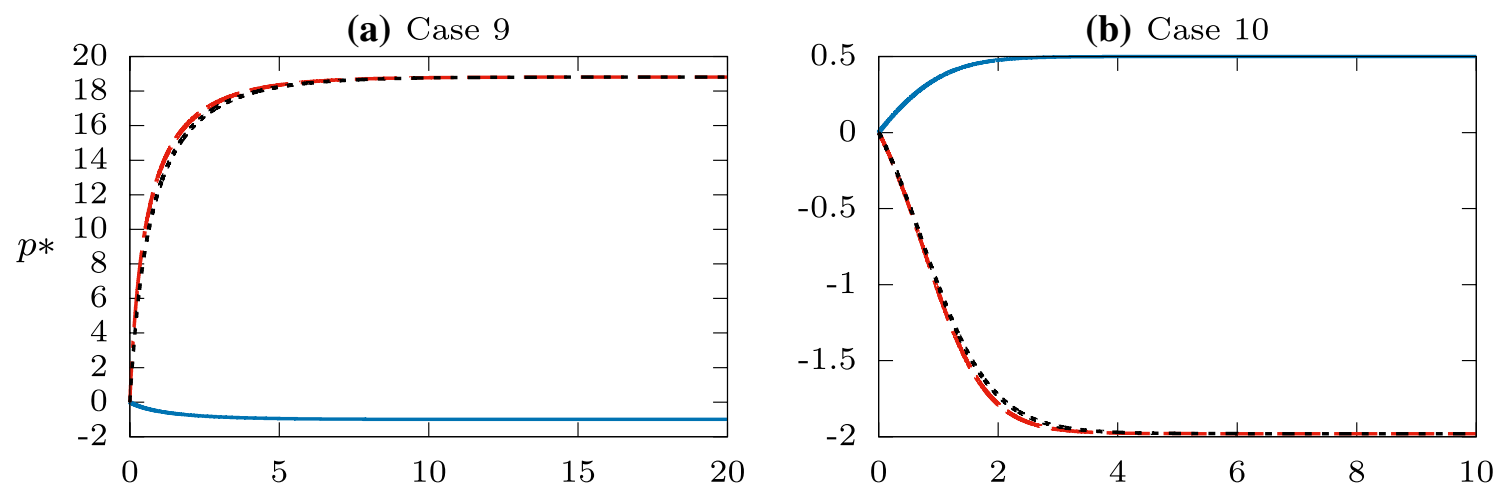

(c) Case 11
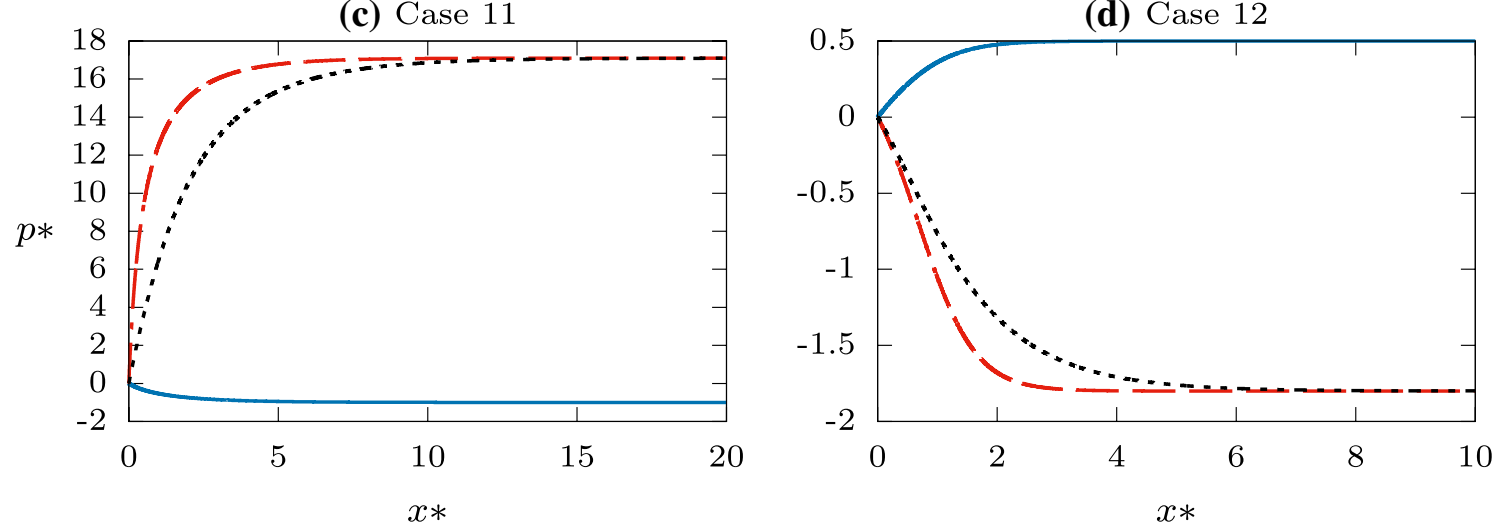

Fig. 6 Comparison between the pressure predictions of different models at $C=10.0$ : DG (blue solid lines); 2WCNP (red dashed lines); 2 WC (black dotted lines)

$R 1=\frac{\alpha_{0}{ }^{2}\left(1-\alpha_{0}\right)}{A}\left(\frac{c_{1}}{u_{p}{ }^{*}}+\frac{c_{2}}{\left(u_{p}{ }^{*}-\alpha_{0}\right)}+\frac{c_{3}}{\left(u_{p}{ }^{*}-\alpha_{0}-D\right)}\right) . \quad-c_{1}\left(\frac{2 \alpha_{0}+D}{\alpha_{0}}\right)-c_{2}\left(\frac{\alpha_{0}+D}{\alpha_{0}}\right)-c_{3}=0$.

Solving the second order terms of Eq. (B13) gives

Using Eqs. (B11) and (B8), the coefficients $c_{1}, c_{2}$ and $c_{3}$, are related as

$c_{1}+c_{2}+c_{3}=0$.

$c_{1}\left(u_{p}{ }^{*}-\alpha_{0}\right)\left(u_{p}{ }^{*}-\alpha_{0}-D\right)+c_{2}\left(u_{p}{ }^{*}-\alpha_{0}-D\right) u_{p}{ }^{*}+c_{3}\left(u_{p}{ }^{*}-\alpha_{0}\right) u_{p}{ }^{*}=1$,

which can be written as

Adding Eq. (B16) to Eq. (B17) gives

$c_{1}\left(u_{p}{ }^{2}-u_{p}{ }^{*}\left(\alpha_{0}+D\right)-\alpha_{0} u_{p}^{*}+\alpha_{0}\left(\alpha_{0}+D\right)\right)+c_{2}\left(u_{p}^{* 2}-u_{p}^{*}\left(\alpha_{0}+D\right)\right)+c_{3}\left(u_{p}^{* 2}-\alpha_{0} u_{p}^{*}\right)=1$

Solving the leading order terms of Eq. (B13) gives

$c_{1}=\frac{1}{\alpha_{0}\left(\alpha_{0}+D\right)}$

Solving the first order terms of Eq. (B13) gives

$-c_{1}\left(2 \alpha_{0}+D\right)-c_{2}\left(\alpha_{0}+D\right)-c_{3} \alpha_{0}=0$.

$c_{1}-c_{1}\left(\frac{2 \alpha_{0}+D}{\alpha_{0}}\right)+c_{2}-c_{2}\left(\frac{\alpha_{0}+D}{\alpha_{0}}\right)=0$

which can be manipulated as

$c_{1}\left(1-\frac{2 \alpha_{0}+D}{\alpha_{0}}\right)+c_{2}\left(1-\frac{\alpha_{0}+D}{\alpha_{0}}\right)=0$,

Divide Eq. (B15) by $\alpha_{0}$ 
$c_{1}\left(\frac{\alpha_{0}}{\alpha_{0}}-\frac{2 \alpha_{0}+D}{\alpha_{0}}\right)+c_{2}\left(\frac{\alpha_{0}}{\alpha_{0}}-\frac{\alpha_{0}+D}{\alpha_{0}}\right)=0$,

$c_{1}\left(\frac{\alpha_{0}+D}{\alpha_{0}}\right)+c_{2}\left(\frac{D}{\alpha_{0}}\right)=0$

$c_{1}\left(\alpha_{0}+D\right)+c_{2} D=0$

$c_{2}=\frac{-c_{1}\left(\alpha_{0}+D\right)}{D}$.

Substituting $c_{1}$ from Eq. (B14) into Eq. (B23) results in

$c_{2}=\frac{-1}{\alpha_{0} D}$.

To obtain $c_{3}$ substitute Eqs. (B24) and (B14) into Eq. (B17) as

$c_{3}=-\frac{1}{\alpha_{0}\left(\alpha_{0}+D\right)}+\frac{1}{\alpha_{0} D}$

which results in

$c_{3}=\frac{1}{\left(\alpha_{0}+D\right) D}$.

Now, substitute Eqs. (B14), (B24) and (B26) into Eq. (B11) as

$$
\begin{aligned}
R_{1}= & \left(\frac{1}{\alpha_{0}\left(\alpha_{0}+D\right) u_{p}{ }^{*}}-\frac{1}{\alpha_{0} D\left(u_{p}{ }^{*}-\alpha_{0}\right)}\right. \\
& \left.+\frac{1}{D\left(\alpha_{0}+D\right)\left(u_{p}{ }^{*}-\alpha_{0}-D\right)}\right) \\
& \times \frac{\alpha_{0}^{2}\left(1-\alpha_{0}\right)}{A} .
\end{aligned}
$$

Substituting Eqs. (8) and (57) into Eq. (45) gives

$$
A=\frac{\alpha_{0}}{\left(1-\alpha_{0}\right) C B^{2}}
$$

which when substituted into Eq. (B27) gives

$$
\begin{aligned}
R_{1} & =\left[\frac{\left(1-\alpha_{0}\right)^{2} C B^{2}}{\left(\alpha_{0}+D\right) u_{p}{ }^{*}}-\frac{\left(1-\alpha_{0}\right)^{2} C B^{2}}{D\left(u_{p}{ }^{*}-\alpha_{0}\right)}\right. \\
& \left.+\frac{\alpha_{0}\left(1-\alpha_{0}\right)^{2} C B^{2}}{\left(u_{p}^{*}-\alpha_{0}-D\right)\left(\alpha_{0}+D\right) D}\right] .
\end{aligned}
$$

Now, using partial fraction decomposition for Eq. (B9) results in $c_{4}\left(u_{p}{ }^{*}-\alpha_{0}-D\right)+c_{5} u_{p}^{*}=1$.

Solving the leading order terms of Eq. (B30) gives

$c_{4}=\frac{-1}{\alpha_{0}+D}$.

Solving the first order terms of Eq. (B30) gives

$c_{4}+c_{5}=0$,

which results in

$c_{5}=-c_{4}=\frac{1}{\alpha_{0}+D}$.

Substitute Eq. (B33) into (B9)

$R_{2}=\frac{D^{2}}{\alpha_{0}+D}\left(-\frac{1}{u_{p}^{*}}+\frac{1}{\left(u_{p}^{*}-\alpha_{0}-D\right)}\right)$.

Now substitute Eqs. (B29) and (B34) into Eq. (B10)

$\left[\frac{\epsilon_{1}}{u_{p}{ }^{*}}+\frac{\epsilon_{2}}{\left(u_{p}{ }^{*}-\alpha_{0}\right)}+\frac{\epsilon_{3}}{\left(u_{p}{ }^{*}-\alpha_{0}-D\right)}+1\right] \mathrm{d} u_{p}{ }^{*}=-\mathrm{d} x^{*}$,

where

$\epsilon_{1}=\frac{\left(1-\alpha_{0}\right)^{2} C B^{2}}{\left(\alpha_{0}+D\right)}-\frac{D^{2}}{\alpha_{0}+D}-\alpha_{0}+D$,

$\epsilon_{2}=\frac{\left(1-\alpha_{0}\right)^{2} C B^{2}}{D}$

$\epsilon_{3}=\frac{\alpha_{0}\left(1-\alpha_{0}\right)^{2} C B^{2}}{\left(\alpha_{0}+D\right) D}+\frac{D^{2}}{\alpha_{0}+D}$.

$\epsilon_{1}$ can be manipulated as

$$
\begin{aligned}
\epsilon_{1} & =\frac{\left(1-\alpha_{0}\right)^{2} C B^{2}}{\left(\alpha_{0}+D\right)}-\frac{D^{2}}{\alpha_{0}+D}+\frac{\left(D-\alpha_{0}\right)\left(\alpha_{0}+D\right)}{\left(\alpha_{0}+D\right)} \\
& =\frac{\left(1-\alpha_{0}\right)^{2} C B^{2}}{\left(\alpha_{0}+D\right)}-\frac{D^{2}}{\alpha_{0}+D}+\frac{D^{2}-\alpha_{0}^{2}}{\left(D+\alpha_{0}\right)} \\
& =\frac{\left(1-\alpha_{0}\right)^{2} C B^{2}}{\left(\alpha_{0}+D\right)}-\frac{\alpha_{0}^{2}}{\alpha_{0}+D} .
\end{aligned}
$$

Using Eq. (54), $\epsilon_{1}$ can be obtained according to Eq. (55) and $\epsilon_{2}$ according to Eq. (56); moreover, $\epsilon_{3}$ can be manipulated as 


$$
\begin{aligned}
\epsilon_{3} & =\frac{\alpha_{0}\left(1-\alpha_{0}\right)^{2} C B^{2}}{\left(\alpha_{0}+D\right) D}+\frac{D^{2}}{\alpha_{0}+D} \\
& =\frac{\alpha_{0}\left(1-\alpha_{0}\right) B C}{\left(\alpha_{0}+D\right)}+\frac{D^{2}}{\alpha_{0}+D} \\
& =\frac{\alpha_{0} D C}{\left(\alpha_{0}+D\right)}+\frac{D^{2}}{\alpha_{0}+D} \\
& =\frac{D\left(\alpha_{0} C+D\right)}{\alpha_{0}+D},
\end{aligned}
$$

which is the same as (57).

To obtain the solution of Eq. (B35), integrate as

$-\epsilon_{1} \ln u_{p}^{*}-\epsilon_{2} \ln \left(u_{p}^{*}-\alpha_{0}\right)-\epsilon_{3} \ln \left(u_{p}^{*}-\alpha_{0}-D\right)-u_{p}^{*}=x^{*}+c$.

Apply the boundary condition, $u_{p}{ }^{*}(0)=1$, to obtain the constant of integration,

$C=-\epsilon_{2} \ln \left(1-\alpha_{0}\right)-\epsilon_{3} \ln \left(1-\alpha_{0}-D\right)-1$,

which when substituted into Eq. (B41) results in the particle phase velocity of the 2WC model as given by Eq. (53).

\section{Appendix C}

In order to derive Eq. (61), substitute $\mathrm{d} u_{p}{ }^{*} / \mathrm{d} x^{*}$, given by Eq. (52), into Eq. (59) as which, after substituting $A$ from Eq. (B28), results in Eq. (61).

\section{References}

1. Balachandar S (2009) A scaling analysis for point-particle approaches to turbulent multiphase flows. Int J Multiph Flow 35(9):801-810

2. Ling Y, Parmar M, Balachandar S (2013) A scaling analysis of added-mass and history forces and their coupling in dispersed multiphase flows. Int J Multiph Flow 57:102-114

3. Alletto M, Breuer M (2012) One-way, two-way and four-way coupled LES predictions of a particle-laden turbulent flow at high mass loading downstream of a confined bluff body. Int J Multiph Flow 45:70-90

4. Ferrante A, Elghobashi S (2003) On the physical mechanisms of two-way coupling in particle-laden isotropic turbulence. Phys Fluids 15(2):315-329

5. Toschi F, Bodenschatz E (2009) Lagrangian properties of particles in turbulence. Annu Rev Fluid Mech 41(1):375-404

6. Slater SA, Young JB (2001) The calculation of inertial particle transport in dilute gas-particle flows. Int J Multiph Flow 27(1):61-87

7. Marble FE (1970) Dynamics of dusty gases. Annu Rev Fluid Mech 2(1):397-446

8. Cantero MI, Balachandar S, Garcia MH (2008) An Eulerian-Eulerian model for gravity currents driven by inertial particles. Int J Multiph Flow 34(5):484-501

9. Fouda YM (2019) Analytical solution of an Eulerian dilute particle-laden flow problem. SN Appl Sci 1:326

10. Guha A (2008) Transport and deposition of particles in turbulent and laminar flow. Annu Rev Fluid Mech 40(1):311-341

11. Drew DA (1983) Mathematical modeling of two-phase flow. Annu Rev Fluid Mech 15(1):261-291

$$
\begin{aligned}
& \frac{\mathrm{d} p^{*}}{\mathrm{~d} u_{p}{ }^{*}} \\
& \quad=-\frac{1}{\alpha_{0}}\left[u_{p}{ }^{*}-\frac{u_{p}{ }^{*}\left(u_{p}{ }^{*}-\alpha_{0}-B\left(1-\alpha_{0}\right)\right)\left(\alpha_{0}{ }^{2}\left(1-\alpha_{0}\right)+A\left(u_{p}{ }^{*}-\alpha_{0}\right)^{3}\right)}{A u_{p}{ }^{*}\left(u_{p}{ }^{*}-\alpha_{0}\right)^{2}\left(u_{p}{ }^{*}-\alpha_{0}-B\left(1-\alpha_{0}\right)\right)}\right] .
\end{aligned}
$$

Then manipulate as

$$
\begin{aligned}
\frac{\mathrm{d} p^{*}}{\mathrm{~d} u_{p}{ }^{*}} & =-\frac{1}{\alpha_{0}}\left(u_{p}{ }^{*}-\frac{\left(\alpha_{0}{ }^{2}\left(1-\alpha_{0}\right)+A\left(u_{p}{ }^{*}-\alpha_{0}\right)^{3}\right)}{A\left(u_{p}{ }^{*}-\alpha_{0}\right)^{2}}\right) \\
& =-\frac{1}{\alpha_{0}}\left(u_{p}{ }^{*}-\frac{\alpha_{0}{ }^{2}\left(1-\alpha_{0}\right)}{A\left(u_{p}{ }^{*}-\alpha_{0}\right)^{2}}-\frac{A\left(u_{p}{ }^{*}-\alpha_{0}\right)^{3}}{A\left(u_{p}{ }^{*}-\alpha_{0}\right)^{2}}\right) \\
& =-\frac{1}{\alpha_{0}}\left(u_{p}{ }^{*}-\frac{\alpha_{0}{ }^{2}\left(1-\alpha_{0}\right)}{A\left(u_{p}{ }^{*}-\alpha_{0}\right)^{2}}-\left(u_{p}{ }^{*}-\alpha_{0}\right)\right) \\
& =-\frac{1}{\alpha_{0}}\left(-\frac{\alpha_{0}{ }^{2}\left(1-\alpha_{0}\right)}{A\left(u_{p}{ }^{*}-\alpha_{0}\right)^{2}}+\alpha_{0}\right),
\end{aligned}
$$

12. Lhuillier D, Chang C-H, Theofanous TG (2013) On the quest for a hyperbolic effective-field model of disperse flows. J Fluid Mech 731:184-194

13. Massoudi M (2010) A mixture theory formulation for hydraulic or pneumatic transport of solid particles. Int J Eng Sci 48(11):1440-1461

14. Drew DA, Passman SL (1999) Theory of multicomponent fluids. Springer, New York

15. Morel C (2015) Mathematical modeling of disperse two-phase flows. Springer, New York

16. Hsu T-J, Jenkins JT, Liu PL-F (2003) On two-phase sediment transport: dilute flow. J Geophys Res 108:3057

17. You R, Peddieson J, Gadiyaram J, Munukutla S (2010) Simulation of particle/fluid flows in vertical circular pipes. Int J Non Linear Mech 45(5):490-506

18. Vellanki NS, Peddieson J, Munukutla S (2006) Application of a stress sharing model to simulation of particle/fluid parallel flow. Acta Mech 181:169-184 
19. Druzhinin OA (1995) On the two-way interaction in two-dimensional particle-laden flows: the accumulation of particles and flow modification. J Fluid Mech 297:49-76

20. Osiptsov AN (1997) Mathematical modeling of dusty-gas boundary layers. Appl Mech Rev 50(6):357-370

21. Foster MR, Duck PW, Hewitt RE (2006) Boundary layers in a dilute particle suspension. Proc R Soc London, Ser A 462(2068):1145-1166

22. Fchaudhary JP, Singh LP (2019) Riemann problem and elementary wave interactions in dusty gas. Appl Math Comput 342:147-165

23. Ku X, Lin J, Sint Annaland M, Hagmeijer R (2016) Numerical simulation of the accumulation of heavy particles in a circular bounded vortex flow. Int J Multiph Flow 87:80-89
24. Ku X, Li H, Lin J, Jin H (2019) Accumulation of heavy particles in circular bounded vortex flows induced by two small rotating cylinders. Int J Multiph Flow 113:71-88

25. Ku X, Li H, Lin J, Jin H, Shen F (2018) Accumulation of heavy particles in a circular bounded vortex flow: Parameter influence and comparison. Int J Multiph Flow 103:124-140

Publisher's Note Springer Nature remains neutral with regard to jurisdictional claims in published maps and institutional affiliations. 